Journal of Animal and Veterinary Advances 11 (11): 1876-1880, 2012

ISSN: $1680-5593$

(C) Medwell Journals, 2012

\title{
Promoter Characterization and Expression Pattern Analysis of Porcine TCAP Gene
}

\author{
Mu Qiao, Huayu Wu, Jingshu Huang, Xianwen Peng, Guisheng Liu, Zheng Feng and Shuqi Mei \\ Hubei Key Laboratory of Animal Embryo and Molecular Breeding, \\ Hubei Academy of Agricultural Sciences, Institute of Animal Husbandry and Veterinary, \\ 430064 Wuhan, P.R. China
}

\begin{abstract}
TCAP is one of the titin interacting Z-disc proteins involved in the regulation and development of normal sarcomeric structure. In this study, researchers cloned the 5 ' upstream region of porcine TCAP gene. Bioinformatic analysis of the $5^{\prime}$ regulatory region has revealed that in addition to several ubiquitous transcription factors binding sites (SP1, AP1 and C/EBP) were found, several putative muscle-specific transcription factor binding sites (MyoD, MyoG and MEF2) were present in this region. Tissue expression analysis showed that the porcine TCAP gene was expressed abundantly in skeletal muscle. The study suggested that TCAP gene might be a prospective candidate gene affecting muscle mass and meat quality traits in the pig and would lay the groundwork for the further investigations on the regulation and physiological function of porcine TCAP gene.
\end{abstract}

Key words: Porcine, TCAP, promoter, bioinformatics, China

\section{INTRODUCTION}

TCAP is one of the titin interacting Z-disc proteins involved in the regulation and development of normal sarcomeric structure (Mason et al., 1999). Mutations in the TCAP were associated with the seventh form of autosomal recessive limb-girdle muscular dystrophy, termed Limb-Girdle Muscular Dystrophy type 2G (LGMD2G) (Moreira et al., 2000). Patients with TCAP mutations develop a marked weakness in the distal muscles of the legs with proximal involvement and most patients lose the ability to walk by the third or fourth decade of life (Moreira et al., 2000).

TCAP has also been shown to interact with and regulate the secretion of Myostatin (MSTN), a negative regulator of muscle growth that inhibits both cell proliferation and differentiation (Nicholas et al., 2002). Knockdown of TCAP by RNA interference in $\mathrm{C}_{2} \mathrm{Cl}_{2}$ myoblast cells inhibits myoblast differentiation and impairs muscle cell growth (Markert et al., 2008). Given the important role of TCAP in myoblast profiferation and differentiation, it has been proposed that TCAP might offer a new therapeutic target for muscular dystrophies. Hitherto, a lot of studies about TCAP are carried out in human and mice, relatively little is know concerning the porcine TCAP gene. In this study, researchers isolated partial sequences of porcine TCAP gene promoter and did some correlated bioinformation analysis. Researchers also, analyzed its tissue expression pattern. These would lay the foundation for further investigations on the regulation and physiological function of porcine TCAP gene.

\section{MATERIALS AND METHODS}

Animals and sample preparation: Three adult Yorkshire pigs were slaughtered. Blood samples were collected immediately after the slaughter of pigs for genomic DNA extraction using phenol/chloroform method (Qiao et al., 2010). Ten different tissues including heart, liver, spleen, lung, kidney, stomach, skeletal muscle, uterus, ovary and adipose tissues were collected then immediately frozen in liquid nitrogen and stored at $-80^{\circ} \mathrm{C}$ for spatial expression analysis. Total RNA was extracted from different tissues with a Trizol reagent (Invitrogen). In case the samples were seriously contaminated with genomic DNA, DNase I (Takara) treatment on the total RNA was carried out before first-strand cDNA synthesis. Reverse transcription was performed as described before (Qiao et al., 2010).

Isolation of the porcine TCAP promoter: According to contrast the genome sequence of porcine TCAP gene (GenBank Accession No.: CU640399.2) and the procine genome, researchers design the primers with primer 5.0.

Corresponding Author: Shuqi Mei, Hubei Key Laboratory of Animal Embryo and Molecular Breeding, Hubei Academy of Agricultural Sciences, Institute of Animal Husbandry and Veterinary, 430064 Wuhan, P.R. China 


\begin{abstract}
The primers were forward: 5'CCTGGGAGGGAAGGTCAA-3'; reverse: 5'CTGGGCTTTGTGCTCTGTC-3'. PCR reactions were carried out in a total volume of $25 \mu \mathrm{L}$ containing $1 \mu \mathrm{L}$ (50 ng) of DNA as template, $0.5 \mu \mathrm{L}$ of each primer $(5 \mu \mathrm{M})$, $2.0 \mu \mathrm{L}$ of each dNTP $(2 \mathrm{mM}), 2.5 \mu \mathrm{L}$ of $10 \times \mathrm{PCR}$ buffer, $2.0 \mu \mathrm{L}$ of $\left(25 \mathrm{mmol} \mathrm{L}^{-1}\right) \mathrm{Mg}^{2+}$ and $1 \mu \mathrm{L}\left(1 \mathrm{U} \mathrm{HL}^{-1}\right)$ of Taq DNA polymerase and $15.5 \mu \mathrm{L}$ sterile water. The PCR amplification profiles were as follows: $94^{\circ} \mathrm{C}$ initial denaturation for $4 \mathrm{~min}, 35$ cycles of $94^{\circ} \mathrm{C}$ denaturation for $45 \mathrm{sec}, 62^{\circ} \mathrm{C}$ annealing for $40 \mathrm{sec}$ and $72^{\circ} \mathrm{C}$ extension for $90 \mathrm{sec}$ followed by a final extension at $72^{\circ} \mathrm{C}$ for $10 \mathrm{~min}$.
\end{abstract}

Expression pattern analysis of the porcine $T C A P$ gene: To check the relative expression level of TCAP mRNA in various porcine tissues, semi-quantitative Reverse Transcription (RT)-PCR was performed as described before (Qiao et al., 2010). The house-keeping gene $G A P D H$ was used as the internal control for determination of target mRNA profiles. The control primers were forward: 5'-ACCACAGTCCATGCCATCAC-3'; reverse: 5'-TCCACCACCCTGTTGCTGTA-3'. The TCAP specific primer pair were forward: 5'-GCTGGTGATGCGAATGGG3'; reverse: 5'-AGACAGGGAGCGACGAAGG-3'. The PCR reaction was carried out with the following cycling parameters: $95^{\circ} \mathrm{C}$ initial denaturation for $4 \mathrm{~min}, 28$ cycles of $95^{\circ} \mathrm{C}$ denaturation for $40 \mathrm{sec}, 58^{\circ} \mathrm{C}$ annealing for $40 \mathrm{sec}$ and $72^{\circ} \mathrm{C}$ extension for $25 \mathrm{sec}$. A final extension was performed at $72^{\circ} \mathrm{C}$ for $7 \mathrm{~min}$.

Bioinformatic analysis: Predictions of putative promoter regions and transcriptional start site were performed with http://www.fruitfly.org/seq_tools/promoter.html (BDGP). The putative transcription factor binding sites were predicted with http://www.cbrc.jp/research/db/ TFSEARCH.html (TFSEARCH) and http://www.cbil. upenn.edu/cgi-bin/tess/tess (TESS) and then chose the sites which got high score in these software. At last, researchers had a comparison with human and the obtained porcine TCAP promoter sequence to select the highly conservative binding sites.

\section{RESULTS AND DISCUSSION}

Characterization of porcine $T C A P$ gene promoter: To investigate the transcriptional regulation of the porcine $T C A P$ gene, the 5 '-flanking region was acquired for further analysis. The product was 1662 and 1466 bp was located in 5'-upper stream of start codon (ATG) (Fig. 1). A comparison with the human TCAP 5'-flanking sequence

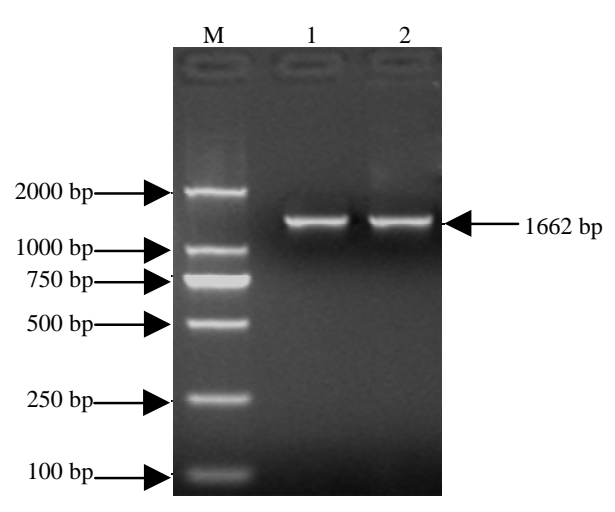

Fig. 1: PCR product of 5 ' upstream of porcine TCAP gene; M: DL2000 DNA Marker; Lanes 1, 2: PCR product

demonstrated a high degree of homology with approximately $76 \%$ nucleotide identity (Fig. 2 ). Sequence analysis indicated that multiple putative binding sites of several ubiquitous transcription factors such as SP1, $\mathrm{AP} 1$ and $\mathrm{C} / \mathrm{EBP}$ were observed in this region. Some reports indicated that transcriptional factor AP1 had transcriptional activation moreover, AP1 binding site existed in gene promoters of multitude inflammatory cell factors and growth factors which prompted that AP1 might take part in the transcriptional regulation of cell factors and growth factors expression (Mao et al., 2012). $\mathrm{C} / \mathrm{EBP}$ together with other Rho factors compose complicated but subtile regulational network. They play an important role on cell multiplication and differentiation, tumorigenesis, organism immunity and stress reaction (Zhu et al., 2009; Wang et al., 2009; Xia et al., 2009).

Several putative muscle-specific transcription factor binding sites (MyoD, MyoG and MEF2) were found within the TCAP promoter. Gene regulation in skeletal muscle is controlled by a family of highly related basic Helix Loop Helix (bHLH) transcription factors, the Myogenic Regulatory Factors (MRFs). The MRF family includes Myf5, MyoD, MyoG and Myf6 (also known as Mrf4).

The MRFs dimerize with E-proteins and bind $\mathrm{E}$ box sequences (CANNTG) in the regulatory regions of muscle genes (Berkes and Tapscott, 2005). The MRFs work in conjunction with multiple isoforms of the MADS-box factors, Mef2a, Mef2c and Mef2d(Blais et al., 2005). Mef2 factors alone do not have myogenic activity but synergize with the MRFs to enhance gene expression during myogenesis (Molkentin et al., 1995; Wang et al., 2001). $\mathrm{MyoD}$ function early in myogenesis to confer a myogenic fate on mesodermal progenitor cells (Rudnicki et al., 1993). MyoG functions later in myogenesis to stimulate 


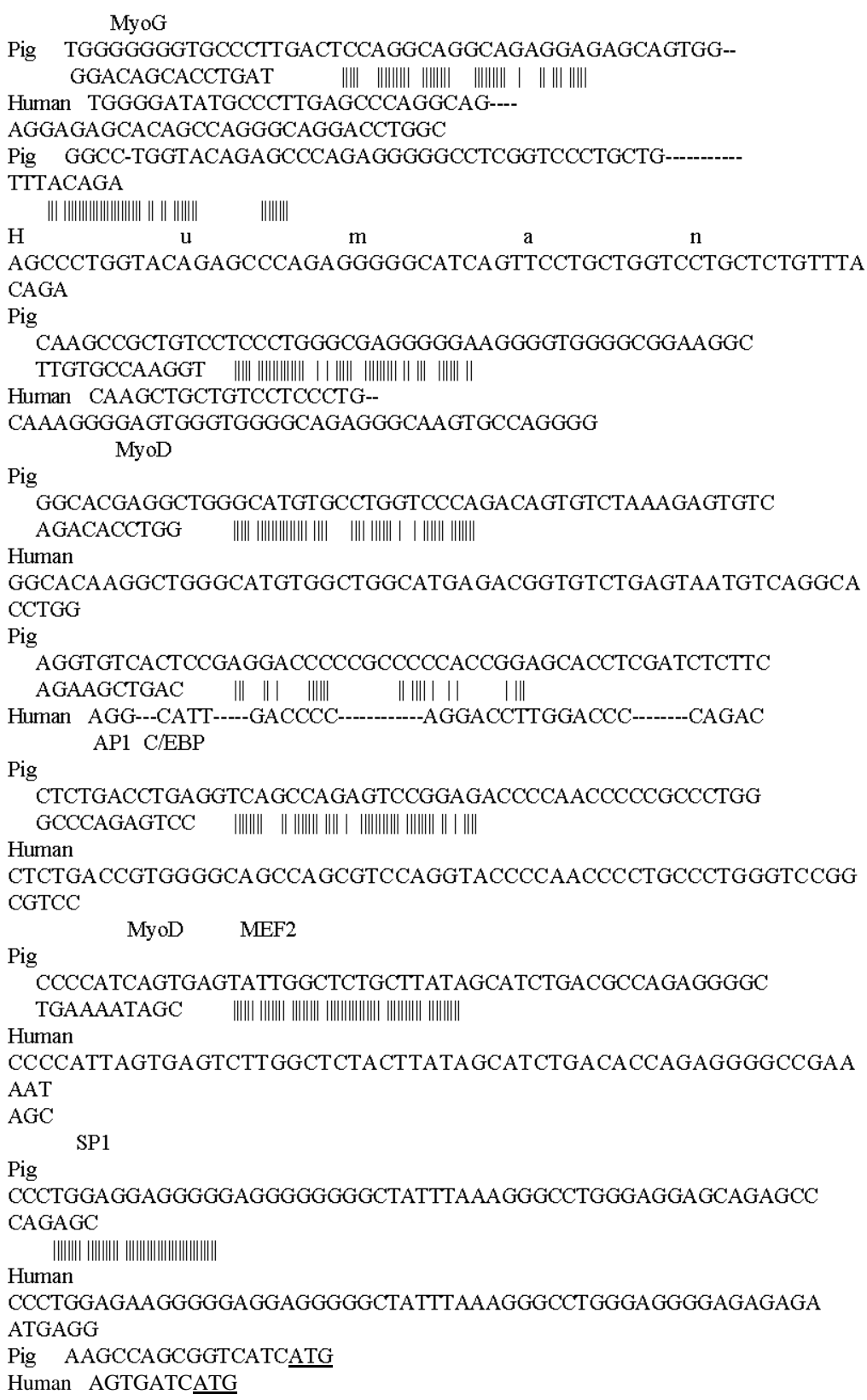

Fig. 2: Contrast of the porcine and human $T C A P$ gene promoter and some binding sites of transcription factors

specified myoblasts to differentiate into functional myofibers (Venuti et al., 1995). Previous research reported that $\mathrm{MyoD}$ play an important role in activating TCAP expression through the promoter proximal $\mathrm{E}$ box and the MyoG is required for normal expression in vivo and physically binds to the TCAP promoter during embryogenesis (Zhang et al., 2011). In cultured skeletal muscle cells, TCAP knockdown resulted in a marked decrease in the expression of MyoD and MyoG, suggesting a refulatory role of TCAP during muscle growth (Markert et al., 2008).

Tissue expression pattern of porcine TCAP: Semiquantitative RT-PCR was carried out to study the relative gene expression level of TCAP in ten various tissues (Fig. 3). The relatively high expression level was found in muscle, heart and kidney, a decreased in adipose tissues and lung, a lower in spleen and liver and had no 


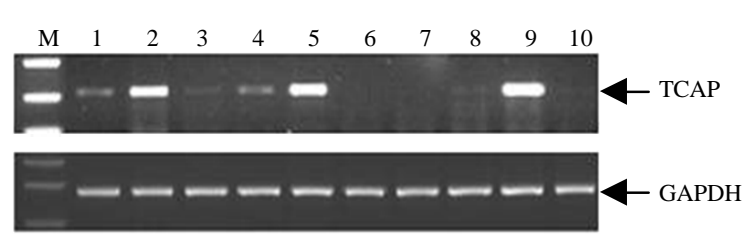

Fig. 3: Tissue expression profile of porcine TCAP gene; 1 : lung; 2: kidney; 3 : liver; 4: adipose tissue; 5 : skeletal muscle; 6 : stomach; 7: ovary; 8: spleen; 9: heart; 10: uterus. The GAPDH expression level is used for the internal control

expression in uterus, ovary and stomach which were consistent with previous studies (Valle et al., 1997). TCAP is a Z-disc associated protein that is thought to be involved in the regulation and development of normal sarcomeric structure. TCAP co-localizes with titin, the interaction with titin is critical for sarcomeric integrity (Gregorio et al., 1998). In this research, it was also shown that TCAP is localized within the Z-line region of adult striated skeletal and cardiac muscles. In the heart, TCAP is necessary for the cardiomyocyte's stretch sensor and the structural organization of the cardiac sarcomere (Bos et al., 2006; Hayashi et al., 2004).

The studies in cattle indicated that TCAP was highly expressed in striated muscle and was developmentally regulated (Mason et al., 1999; Valle et al., 1997). The over-expression of TCAP in $\mathrm{C} 2 \mathrm{C} 12$ cell or primary cardiac myocyte disrupted the process of myofibril, suggesting its requirement for sarcomerogenesis in myocytes (Gregorio et al., 1998). All these evidences suggested that TCAP might have similar biological function on muscle growth and meat quality traits in the pig.

\section{CONCLUSION}

Researchers have isolated and characterized partial porcine TCAP gene promoter and found several putative muscle-specific transcription factor binding sites such as MyoD, MyoG and MEF2. The study would lay the foundation for future studies on porcine TCAP function and the regulatory mechanism in porcine skeletal muscle. In addition, the tissues expression profiles of pig TCAP mRNA displayed its abundant expression in skeletal muscle. The results implied that TCAP could be considered as a potential candidate for porcine muscle growth and meat quality.

\section{ACKNOWLEDGEMENTS}

This study was supported financially by the National Natural Science Foundation of China (30901 025), Hubei
Key Laboratory of Animal Embryo and Molecular Breeding, Institute of Animal Husbandry and Veterinary, Hubei Academy of Agricultural Sciences, 430064 Wuhan, China (2012ZD143).

\section{REFERENCES}

Berkes, C.A. and S.J. Tapscott, 2005. MyoD and the transcriptional control of myogenesis. Semin. Cell Dev. Biol., 16: 585-595.

Blais, A., M. Tsikitis, D. Acosta-Alvear, R. Sharan, Y. Kluger and B.D. Dynlacht, 2005. An initial blueprint for myogenic differentiation. Genes Dev., 19: 553-569.

Bos, J.M., R.N. Poley, M. Ny, D.J. Tester and X. Xu et al., 2006. Genotype-phenotype relationships involving hypertrophic cardiomyopathy-associated mutations in titin, muscle LIM protein and telethonin. Mol. Genet. Metab., 88: 78-85.

Gregorio, C.C., K. Trombitas, T. Centner, B. Kolmerer and G. Stier et al., 1998. The $\mathrm{NH}_{2}$ terminus of titin spans the z-disc: Its interaction with a novel 19-kd ligand (t-cap) is required for sarcomeric integrity. J. Cell Biol., 143: 1013-1027.

Hayashi, T., T. Arimura, M. Itoh-Satoh, K. Ueda and S. Hohda et al., 2004. Tcap gene mutations in hypertrophic cardiomyopathy and dilated cardiomyopathy. J. Am. Coll. Cardiol., 44: 2192-2201.

Mao, L.L., Y.L. Pan, X.Y. Wan, X.M. Wang and $\mathrm{K}$. Feng et al., 2012. Cloning and sequence analysis of rabbit interleukin-10 (IL-10) gene promoter. J. Anim. Vet. Adv., 11: 323-326.

Markert, C.D., J. Ning, J.T. Staley, L. Heinzke and C.K. Childers et al., 2008. TCAP knockdown by RNA interference inhibits myoblast differentiation in cultured skeletal muscle cells. Neuromuscul. Disord., 18: 413-422.

Mason, P., S. Bayol and P.T. Loughna, 1999. The novel sarcomeric protein telethonin exhibits developmental and functional regulation. Biochem. Biophys. Res. Commun., 257: 699-703.

Molkentin, J.D., B.L. Black, J.F. Martin and E.N. Olson, 1995. Cooperative activation of muscle gene expression by MEF2 and myogenic bHLH proteins. Cell, 83: 1125-1136.

Moreira, E.S., T.J. Wiltshire, G. Faulkner, A. Nilforoushan and M. Vainzof et al., 2000. Limb-girdle muscular dystrophy type $2 \mathrm{G}$ is caused by mutations in the gene encoding the sarcomeric protein telethonin. Nat. Genet., 24: 163-166. 
Nicholas, G., M. Thomas, B. Langley, W. Somers and K. Patel et al., 2002. Titin-cap associates with and regulates secretion of, myostatin. J. Cell. Physiol., 193: $120-131$

Qiao, M., H.Y. Wu, F.E. Li, S.W. Jiang, Y.Z. Xiong and C.Y. Deng, 2010. Molecular characterization, expression profile and association analysis with carcass traits of porcine $L C A T$ gene. Mol. Biol. Rep., 37: 2227-2234.

Rudnicki, M.A., P.N.J. Schnegelsberg, R.H. Stead, T. Braun, H.H. Arnold and R. Jaenisch, 1993. Myod or myf-5 is required for the formation of skeletal muscle. Cell, 75: 1351-1359.

Valle, G., G. Faulkner, A. de Antoni, B. Pacchioni and A. Pallavicini et al., 1997. Telethonin, a novel sarcomeric protein of heart and skeletal muscle. FEBS Lett., 415: 163-168.

Venuti, J.M., J.H. Morris, J.L. Vivian, E.N. Olson and W.H. Klein, 1995. Myogenin is required for late but not early aspects of myogenesis during mouse development. J. Cell. Biol., 128: 563-576.
Wang, D.Z., M.R. Valdez, J. McAnally, J. Richardson and E.N. Olson, 2001. The MEF2C gene is a direct transcriptional target of myogenic bHLH and MEF2 proteins during skeletal muscle development. Development, 128: 4623-4633.

Wang, H.Z., Y. Wang, D.Q. Sun and D.G. Liu, 2009. Molecular mechanism of tumor suppression activity of C/EBPâ mRNA 3'UTR: Simultaneous deletion of 3 short sequences in the $3^{\prime}$ UTR reduces its tumor suppression activity. Prog. Biochem. Biophys., 36: 1134-1140.

Xia, W., H. Hu, Z. Wei-nan, P. Ze-min, J. Xu-peng, Z. Dong-Hui and P. Ze-min, 2009. The research progress of nuclear transcription factor $\mathrm{C} / \mathrm{EBP} \beta$. Prog. Mod. Biomed., 9: 3198-3200.

Zhang, S., P. Londhe, M. Zhang and J.K. Davie, 2011. Transcriptional analysis of the titin cap gene. Mol. Genet. Genomics, 285: 261-272.

Zhu,.X.D., W.L. Feng and Z.G. Huang, 2009. Effect of enhancer-binding protein $\mathrm{C} / \mathrm{EBP} \alpha$ on differentiation and apoptosis of K562 cells and its possible mechanism. Tumor, 29: 220-225. 